BMJ Open Sport \& Exercise Medicine

\section{Applied sport science and medicine of women's rugby codes: a systematic- scoping review and consensus on future research priorities protocol}

\begin{abstract}
To cite: Heyward 0 Emmonds S, Roe G, et al. Applied sport science and medicine of women's rugby codes: a systematic-scoping review and consensus on future research priorities protocol. BMJ Open Sport \& Exercise Medicine 2021;7:e001108. doi:10.1136/ bmjsem-2021-001108
\end{abstract}

Accepted 16 July 2021

Check for updates

(C) Author(s) (or their employer(s)) 2021. Re-use permitted under CC BY-NC. No commercial re-use. See rights and permissions. Published by BMJ.

${ }^{1}$ Carnegie Applied Rugby Research (CARR) centre, Carnegie School of Sport, Leeds Beckett University, Leeds, UK

${ }^{2}$ Rugby Football Union,

Twickenham, UK

${ }^{3}$ Leeds Rhinos Rugby League club, Leeds, UK

${ }^{4}$ England Performance Unit, Rugby Football League, Leeds, UK

${ }^{5}$ Bath Rugby, Bath, UK

${ }^{6}$ Department for Health, University of Bath, Bath, UK

${ }^{7}$ School of Science and

Technology, University of New England, Armidale, New South Wales, Australia

${ }^{8}$ Division of Exercise Science and Sports Medicine,

Department of Human Biology, Faculty of Health Sciences,

University of Cape Town and the Sports Science Institute of South Africa, Cape Town, South Africa

Correspondence to Mr Omar Heyward;

o.heyward@leedsbeckett.ac.uk

\section{ABSTRACT}

Women's rugby (rugby league, rugby union and rugby sevens) has recently grown in participation and professionalisation. There is under-representation of women-only cohorts within applied sport science and medicine research and within the women's rugby evidence base. The aims of this article are: Part 1: to undertake a systematic-scoping review of the applied sport science and medicine of women's rugby, and Part 2: to develop a consensus statement on future research priorities. This article will be designed in two parts: Part 1: a systematic-scoping review, and Part 2: a three-round Delphi consensus method. For Part 1, systematic searches of three electronic databases (PubMed (MEDLINE), Scopus, SPORTDiscus (EBSCOhost)) will be performed from the earliest record. These databases will be searched to identify any sport science and medicine themed studies within women's rugby. The Preferred Reporting Items for Systematic Reviews and Meta-analyses extension for Scoping Reviews will be adhered to. Part 2 involves a three-round Delphi consensus method to identify future research priorities. Identified experts in women's rugby will be provided with overall findings from Part 1 to inform decision-making. Participants will then be asked to provide a list of research priority areas. Over the three rounds, priority areas achieving consensus ( $\geq 70 \%$ agreement) will be identified. This study has received institutional ethical approval. When complete, the manuscript will be submitted for publication in a peer-reviewed journal. The findings of this article will have relevance for a wide range of stakeholders in women's rugby, including policymakers and governing bodies.

\section{INTRODUCTION}

Rugby codes (rugby union, rugby league and rugby sevens; herein referred to as 'rugby') are played by men and women at junior, senior, community and elite levels. Rugby match play is characterised by intermittent high-intensity actions and collisions (eg, sprinting, tackling) interspersed with low to moderate intensity actions (eg, walking, jogging). ${ }^{1}$ The popularity and professionalisation of women's rugby has grown dramatically in recent times,

\section{Key messages}

What is already known

- The popularity and professionalism of women's rugby has recently grown dramatically.

- A scoping review is appropriate for the applied sport science and medicine of women's rugby to examine the extent and range of the evidence base, and identify research gaps in the literature.

- The Delphi method has been used widely in sport science and medicine, and offers a scientifically rigorous technique to determine research priorities.

What this study could add

- This will be the first scoping review of applied sport science and medicine research on women's rugby.

- This review will use a range of stakeholders with sports science and medicine expertise to identify research priorities within women's rugby.

- The findings of this article will have relevance and benefit for a wide range of stakeholders in women's rugby, including policymakers and governing bodies.

with participation in rugby league and rugby union growing by $\sim 30 \%$ between 2017 and 2019. ${ }^{23}$ In 2016 at the Rio Olympics, women's rugby sevens was introduced for the first time. ${ }^{4}$ Furthermore, financial investment into both rugby league and rugby union in England was seen in 2017 with the introduction of the Premier 15s (Rugby Football Union) and Women's Super League (Rugby Football League) competitions. Similar trends have been observed worldwide, with the National Rugby League Women's Premiership in Australia introduced in 2018. These developments have contributed to bringing further attention to women's rugby.

As little as $4 \%$ of studies within applied sport science and medicine research include women-only cohorts, demonstrating the need for more research. ${ }^{5}$ Given the disparity between male and female cohorts, there is only a small body of evidence reviewing 
specific topics within women's rugby codes. To the authors' knowledge, this evidence base comprises only three reviews, which have investigated injury incidence in women's rugby league ${ }^{7}$ and women's rugby union, ${ }^{8}$ and match demands, anthropometric and physical qualities in women's rugby sevens. ${ }^{9}$ In contrast, there are several reviews published since 2020 within the applied sport science and medicine of rugby with male-only cohorts. $^{10-18}$

A recent call to action, which proposed further research within women's rugby, was a vital step in supporting sportswomen with an evidence-based framework. ${ }^{19}$ To begin development of an evidence-based framework, a scoping review can be used to understand the current evidence base. Scoping reviews map the broad key themes and identify knowledge gaps of developing research areas ${ }^{20}$ and have recently been undertaken in rugby for determining health outcomes, ${ }^{16}$ and in women's football to understand the quantity of research. ${ }^{21}$ A scoping review is appropriate for the applied sport science and medicine of women's rugby to examine the extent and range of the evidence base, and identify research gaps in the literature. Once the current evidence base has been established, determining the research priorities is then important. Research priorities should be co-constructed from a range of stakeholders with sports science and medicine expertise, to ensure the research has translational impact and benefit. ${ }^{22} 23$

When considering sports science and medicine research for women's rugby, a Delphi method offers a technique to problem solve, generate ideas or determine priorities. ${ }^{24}$ A key strength of this method is that it allows balanced participant participation, which minimises the risk of bias, thus enhancing scientific rigour. ${ }^{24}$ The technique has been used widely within sport science and medicine, for example, to inform return to play after hamstring injury in football, ${ }^{25}$ and to determine consensus on a video analysis framework in rugby. ${ }^{26}$ The purpose of this article is therefore twofold: Part 1: to undertake a systematic-scoping review of the applied sport science and medicine of women's rugby, and Part 2: to develop a consensus statement on future research priorities within women's rugby.

\section{METHODS}

This article will be designed in two parts: Part 1: a systematic-scoping review, ${ }^{27}$ and Part 2: a three-round Delphi consensus method. ${ }^{24}$ 28-30

\section{Part 1: systematic-scoping review}

Search strategy

A systematic search of three electronic databases (PubMed (MEDLINE), Scopus, SPORTDiscus (EBSCOhost)) will be performed from the earliest record available. Using previously published applied sport science and medicine reviews as a guide, ${ }^{181031}$ search terms will be categorised into primary terms of those pertaining to (1) women, (2) rugby, and (3) applied sport science and medicine. Primary terms will be combined using the AND function. Secondary search terms pertaining to women ('female', 'women'), rugby ('rugby league', 'rugby union', 'rugby sevens') and applied sport science and medicine ('performance', 'match', 'characteristics', 'peak', 'game', 'competition', 'skill', 'technical', 'anthropometric', 'composition', 'physical', 'strength', 'power', 'jump', 'speed', 'fitness', 'aerobic', 'training', 'qualities', 'neuro*', 'muscle damage', 'fatigue', 'recovery', 'nutrition', 'injury', 'incidence', 'psychology') will be combined by the OR function. Searches will be performed in title and abstract fields. Searches will be limited to the English language. Reference lists of selected studies will be manually searched for additional eligible papers.

\section{Study selection}

Titles and abstracts identified by the search strategy will be evaluated against the eligibility criteria independently by two authors (OH and SS). The reviewers will meet to discuss the eligibility criteria in depth, and then proceed to assess the titles and abstracts independently. If the reviewers disagree on the eligibility of a study, it will be discussed with a third reviewer (SE/GR/BJ) whose decision will be binding. A flow diagram will be produced to show the selection process, according to the Preferred Reporting Items for Systematic Reviews and Metaanalyses extension for Scoping Reviews and the Preferred Reporting Items for Systematic Reviews and Meta-Analysis Search Extension guidelines. ${ }^{27} 32$

\section{Inclusion criteria}

- Research articles are not limited by geographical location.

- All levels of participation and age.

- Studies investigate women's rugby union, rugby league or rugby sevens.

- Studies investigate women's rugby as a sport.

- Studies investigate the applied sport science of women's rugby from a performance perspective.

- Studies investigate injury epidemiology within women's rugby.

- Peer-reviewed original research studies.

\section{Exclusion criteria}

- Non-English language studies.

- Review articles, conference proceedings, editorials, case studies, theses, grey literature.

- Studies where outcome measures do not differentiate between sex.

- Studies that do not differentiate outcome measures between rugby and other sports.

- Studies that use women's rugby players as participants to investigate a broader concept, but do not inform day-to-day practice or have minimal direct application to women's rugby (eg, strength imbalance in team sports).

- Sex of participants not specified.

- Male participants only. 


\section{Data charting}

Data will be extracted and assimilated as per best practice methodology. ${ }^{20} 27$ Studies will be categorised into sport science and medicine themes, determined by primary aims and outcome measures. Similar methods have been used in a recent scoping review. ${ }^{21}$ Data charting will be performed by the lead author $(\mathrm{OH})$ and cross-checked by a second (SS) using a predeveloped charting sheet. Any disagreements will be discussed by the research team. The following data will be extracted:

1. Author (s).

2. Year of publication.

3. Country of origin.

4. Code of rugby.

5. Study cohort and sample size.

6. Participant characteristics (age, height, body mass).

7. Study aim.

8. Study outcome measures.

9. Intervention time course and comparator (if applicable).

10. Injury definition (if applicable).

11. Number of matches (if applicable).

12. Key findings.

If unforeseen additional useful data can be charted, then further categories may also be included, or table headings adjusted on the data charting form. Where study full texts cannot be retrieved, attempts to obtain the paper will be made via the university library. Where these attempts are unsuccessful, the corresponding author will be contacted to request a copy. If full text cannot be obtained, the paper will be excluded.

\section{Part 2: Delphi technique}

\section{Participants}

To identify research priority areas within female rugby this project aims to recruit a selection of experts. Participants will include a group of researchers, professionals or players who have experience or affiliation to women's rugby. Consideration will be given to ensure equal representation by experts in sport science (eg, applied sport scientist), medicine (eg, chief medical officer) or governance (eg, director of women's rugby). ${ }^{28} 33$ This will be achieved by recruiting an equal number of participants from each discipline. For players to be included within the expert panel they must meet the following criteria: (1) played internationally and (2) actively involved in sport science or medicine (eg, PhD (candidate), lecturer, medical doctor). Although the inclusion criteria for players may limit the number of participants, as per sampling guidelines, it ensures the possibility of players drawing clear inferences and conclusions from the data. ${ }^{34}$ All participants will be recruited via a purposeful sampling technique, which involves selecting knowledgeable individuals with specific experience in women's rugby. ${ }^{35}$ A sample size of $11-25$ is typical for a the final round of a Delphi study. ${ }^{29}$ To account for dropout and non-responders, a large sample $(>50)$ of potential participants will be contacted to partake in this study.

\section{Round 1}

In the first round of questioning, the expert panel will be provided with, and asked to read, the main findings from Part 1 (overall results from the included studies) in order to inform them on the current sports science and medicine research in women's rugby. ${ }^{24}{ }^{28}$ Using both the main findings from Part 1 and the participants' own experiences, the expert panel will be asked to provide a list of future research areas that they believe to be important. Results from the first round will be grouped by means of thematic analysis, after duplicates are removed. ${ }^{36}$ This process involves an abstraction process whereby expert-identified priority research areas will be allocated to subthemes to develop main themes. ${ }^{36}$ Data will be obtained via Qualtrics online software (Qualtrics, Provo, USA).

\section{Round 2}

The expert panel will receive the list of priority research areas developed in round 1. Experts will then be required to: (1) rate future research priority of each of the applied sport science and medicine areas from low to high on a 5-point agreement Likert scale (1: very low priority, 2: low priority, 3: medium priority, 4: high priority, 5: very high priority). Panellists will again be provided an opportunity to include any additional sport science and medicine areas that they think are a research priority. Following the completion of rating by each expert, the results will be analysed.

\section{Round 3}

The expert panel will receive feedback on round 2 in the form of descriptive statistics, which will enable reflection before expressing their final opinion. Panellists will be asked to rerate (using the same 5-point Likert scale) the criteria from round 2 that did not reach consensus, including any areas that panel members included in round 2. When assessing consensus, Likert scale ratings will be combined (ie, very low and low priority, and high and very high priority will be grouped). Consensus will be deemed to have been reached if $\geq 70 \%$ of panellists gave the same rating. ${ }^{29} 3337$ As the aim of this Delphi method is to ascertain the research priority of all applied sport science and medicine areas, no areas will be removed on the grounds of low priority. Research areas that do not reach consensus after round 3 will still be reported as they may be important to some stakeholder groups but not to all.

Agreement ratings will be obtained separately for each research area. For each area, the mean agreement $( \pm \mathrm{SD})$ will be calculated.

\section{DISCUSSION}

To the authors' knowledge, this will be the first scoping review of applied sport science and medicine research on women's rugby. This review will identify broad themes that have been addressed in the evidence base as well as providing consensus on future research priorities, thus 
providing alignment and direction for future research. The findings of this article will have relevance for a wide range of stakeholders in women's rugby, including policymakers and governing bodies.

\section{ETHICS AND DISSEMINATION}

This study has received ethics approval from Leeds Beckett University Research Ethics Committee. When completed, the findings of the manuscript will be presented in a variety of formats. They will be submitted for review and publication in a peer-reviewed sport and exercise medicine journal and submitted to be presented at conferences (where applicable). To enhance the translational impact of the manuscript, multimedia resources will be created with the aim of disseminating key findings via other channels (eg, social media platforms). ${ }^{38}$

Twitter 0mar Heyward @0mar_Heyward, Stacey Emmonds @S_Emmonds, Gregory Roe @GregoryRoe, Sean Scantlebury @SeanScants, Keith Stokes @ drkeithstokes and Ben Jones @23benjones

Contributors $\mathrm{OH}, \mathrm{KS}$ and $\mathrm{BJ}$ conceptualised the manuscript. $\mathrm{OH}, \mathrm{SE}, \mathrm{GR}$ and $\mathrm{BJ}$ identified the method and framework for the manuscript. $\mathrm{OH}$ and SS proposed the search strategy. $\mathrm{OH}$ prepared the manuscript. All authors contributed to subsequent drafts and approved the final version.

Funding The authors have not declared a specific grant for this research from any funding agency in the public, commercial or not-for-profit sectors.

Competing interests None declared.

Patient and public involvement Patients and/or the public were involved in the design, or conduct, or reporting, or dissemination plans of this research. Refer to the Methods section for further details.

Patient consent for publication Not required.

Provenance and peer review Not commissioned; externally peer reviewed. Data availability statement All data relevant to the study are included in the article.

Open access This is an open access article distributed in accordance with the Creative Commons Attribution Non Commercial (CC BY-NC 4.0) license, which permits others to distribute, remix, adapt, build upon this work non-commercially, and license their derivative works on different terms, provided the original work is properly cited, appropriate credit is given, any changes made indicated, and the use is non-commercial. See: http://creativecommons.org/licenses/by-nc/4.0/.

\section{ORCID iDs}

Omar Heyward http://orcid.org/0000-0002-7390-6511

Ben Jones http://orcid.org/0000-0002-4274-6236

\section{REFERENCES}

1 Owen C, Till K, Weakley J, et al. Testing methods and physica qualities of male age grade rugby union players: a systematic review. PLoS One 2020;15:e0233796.

2 British Broadcasting Corporation. World Rugby launches campaign to increase participation in women's game, 2019. Available: https:// www.bbc.co.uk/sport/rugby-union/48348245 [Accessed 17 Jun 2020].

3 Rugby Football League. Rugby football League welcomes 30 per cent growth in female teams, 2018. Available: https://www.rugbyleague.com/article/51990/rugby-football-league-welcomes-per-centgrowth-in-female-teams [Accessed 10 Dec 2018].

4 International Olympic Committee. Rugby: history of rugby at the Olympic Games 2017.

5 Costello JT, Bieuzen F, Bleakley CM. Where are all the female participants in sports and exercise medicine research? Eur $J$ Sport Sci 2014;14:847-51.

6 Emmonds S, Heyward O, Jones B. The challenge of applying and undertaking research in female sport. Sports Med Open 2019;5:51.
7 King DA, Hume PA, Milburn PD, et al. Match and training injuries in rugby League: a review of published studies. Sports Med 2010;40:163-78.

8 King D, Hume P, Cummins C, et al. Match and training injuries in women's rugby Union: a systematic review of published studies. Sports Med 2019;49:1559-74.

9 Sella FS, McMaster DT, Beaven CM, et al. Match demands, anthropometric characteristics, and physical qualities of female rugby Sevens athletes: a systematic review. J Strength Cond Res 2019;33:3463-74.

10 Till K, Weakley J, Read DB, et al. Applied sport science for male Age-Grade rugby union in England. Sports Med Open 2020;6:14.

11 Kelly VG, Oliver LS, Bowtell J, et al. Inside the belly of a beast: Individualizing nutrition for young, professional male rugby League players: a review. Int J Sport Nutr Exerc Metab 2020;31:73-89.

12 Colomer CME, Pyne DB, Mooney M, et al. Performance analysis in rugby Union: a critical systematic review. Sports Med Open 2020;6:4.

13 Brazier J, Antrobus M, Stebbings GK, et al. Anthropometric and physiological characteristics of elite male rugby athletes. J Strength Cond Res 2020;34:1790-801.

14 West SW, Starling L, Kemp S, et al. Trends in match injury risk in professional male rugby Union: a 16-season review of 10 851 match injuries in the English Premiership (2002-2019): the professional rugby injury surveillance project. $\mathrm{Br} J$ Sports Med 2021;55:676-82.

15 Naughton M, Jones B, Hendricks S. Quantifying the collision dose in rugby League: a systematic review, meta-analysis, and critical analysis. Sports Med - Open 2020;6.

16 Griffin SA, Panagodage Perera NK, Murray A, et al. The relationships between rugby union, and health and well-being: a scoping review. Br J Sports Med 2021;55:1-11.

17 Dalton-Barron N, Whitehead S, Roe G, et al. Time to embrace the complexity when analysing GPs data? A systematic review of contextual factors on match running in rugby League. J Sports Sci 2020;38:1161-80.

18 Aben HGJ, Hills SP, Cooke CB, et al. Profiling the Post-match recovery response in male rugby. J Strength Cond Res 2020;Publish Ahead of Print.

19 Cummins C, Melinz J, King D, et al. Call to action: a collaborative framework to better support female rugby League players. $\mathrm{Br} J$ Sports Med 2020;54:501-2.

20 Peters MDJ, Godfrey CM, Khalil H, et al. Guidance for conducting systematic scoping reviews. Int J Evid Based Healthc 2015;13:141-6.

21 Okholm Kryger K, Wang A, Mehta R. Research on women's football: a scoping review. Sci Med Footb 2021;00:1-10.

22 Sacristán JA, Aguarón A, Avendaño C, et al. Patient involvement in clinical research: why, when, and how. Patient Prefer Adherence 2016;10:631-40.

23 Boivin A, Currie K, Fervers B, et al. Patient and public involvement in clinical guidelines: international experiences and future perspectives. Qual Saf Health Care 2010;19:e22.

24 McMillan SS, King M, Tully MP. How to use the nominal group and Delphi techniques. Int J Clin Pharm 2016;38:655-62.

25 van der Horst N, Backx F, Goedhart EA, et al. Return to play after hamstring injuries in football (soccer): a worldwide Delphi procedure regarding definition, medical criteria and decision-making. $\mathrm{Br} J$ Sports Med 2017;51:1583-91.

26 Hendricks S, Till K, den Hollander S, et al. Consensus on a video analysis framework of descriptors and definitions by the rugby Union video analysis consensus group. Br J Sports Med 2020:54:566-72.

27 Tricco AC, Lillie E, Zarin W, et al. PRISMA extension for scoping reviews (PRISMA-ScR): checklist and explanation. Ann Intern Med 2018;169:467-73.

28 Shrier I. Consensus statements that fail to recognise dissent are flawed by design: a narrative review with 10 suggested improvements. Br J Sports Med 2020.

29 Diamond IR, Grant RC, Feldman BM, et al. Defining consensus: a systematic review recommends methodologic criteria for reporting of Delphi studies. J Clin Epidemiol 2014;67:401-9.

30 Jünger S, Payne SA, Brine J, et al. Guidance on conducting and reporting Delphi studies (CREDES) in palliative care: recommendations based on a methodological systematic review. Palliat Med 2017:31:684-706.

31 Whitehead S, Weakley J, Cormack S, et al. The applied sports science and medicine of Netball: a systematic scoping review. Sports Med 2021:1-17.

32 Rethlefsen ML, Kirtley S, Waffenschmidt S, et al. PRISMA-S: an extension to the PRISMA statement for reporting literature searches in systematic reviews. Syst Rev 2021;10:39. 
33 Verhagen AP, de Vet HC, de Bie RA, et al. The Delphi list: a criteria list for quality assessment of randomized clinical trials for conducting systematic reviews developed by Delphi consensus. $J$ Clin Epidemiol 1998;51:1235-41.

34 Kemper EA, Springfield S, Teddlie C. Mixed Methods Sampling Strategies in Social Science Research. In: Handbook of Mixed Methods in Social \& Behavioral Research, 2003: 273-96.

35 Palinkas LA, Horwitz SM, Green CA, et al. Purposeful sampling for qualitative data collection and analysis in mixed method implementation research. Adm Policy Ment Health 2015;42:533-44.
36 Crowe M, Inder M, Porter R. Conducting qualitative research in mental health: thematic and content analyses. Aust N Z J Psychiatry 2015;49:616-23.

37 Kleynen M, Braun SM, Bleijlevens MH, et al. Using a Delphi technique to seek consensus regarding definitions, descriptions and classification of terms related to implicit and explicit forms of motor learning. PLoS One 2014;9:e100227.

38 Barton CJ, Merolli MA. It is time to replace publish or perish with get visible or vanish: opportunities where digital and social media can reshape knowledge translation. Br J Sports Med 2019;53:594-8. 